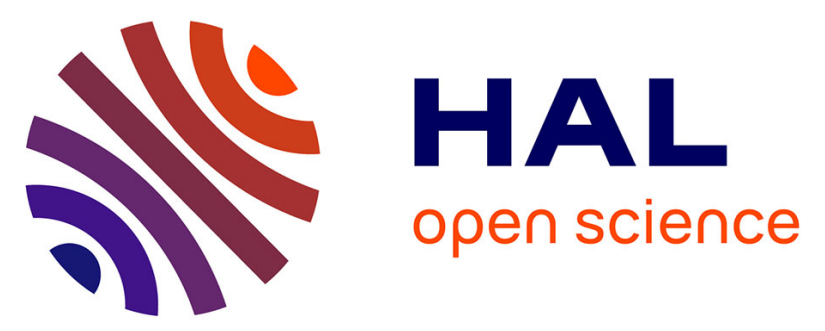

\title{
From Supercontinuum Generation to Carrier Shocks: Extreme Nonlinear Propagation in Photonic Crystal Fiber
}

\author{
J.M. Dudley, B. Kibler, G. Genty, S. Coen, P. Kinsler
}

\section{To cite this version:}

J.M. Dudley, B. Kibler, G. Genty, S. Coen, P. Kinsler. From Supercontinuum Generation to Carrier Shocks: Extreme Nonlinear Propagation in Photonic Crystal Fiber. Conference on Lasers and ElectroOptics/Quantum Electronics and Laser Science Conference, May 2007, Baltimore, United States. pp.2070-2071, 10.1109/QELS.2007.4431340 . hal-00472292

\section{HAL Id: hal-00472292 \\ https://hal.science/hal-00472292}

Submitted on 14 Apr 2021

HAL is a multi-disciplinary open access archive for the deposit and dissemination of scientific research documents, whether they are published or not. The documents may come from teaching and research institutions in France or abroad, or from public or private research centers.
L'archive ouverte pluridisciplinaire HAL, est destinée au dépôt et à la diffusion de documents scientifiques de niveau recherche, publiés ou non, émanant des établissements d'enseignement et de recherche français ou étrangers, des laboratoires publics ou privés. 


\title{
From Supercontinuum Generation to Carrier Shocks: Extreme Nonlinear Propagation in Photonic Crystal Fiber
}

\author{
J. M. Dudley and B. Kibler \\ Institut FEMTO-ST, Département d'Optique P. M. Duffieux, Université de Franche-Comté, 25030 Besançon, France \\ john.dudley@univ-fcomte.fr \\ G. Genty \\ Helsinki University of Technology, Metrology Research Institute, P.O.Box 3000, FIN-02015 HUT, Finland \\ S. Coen \\ Department of Physics, University of Auckland, Private Bag 92019, Auckland, New Zealand \\ P. Kinsler \\ Blackett Laboratory, Imperial College London, SW7 2BW, United Kingdom.
}

\begin{abstract}
We review supercontinuum generation in photonic crystal fiber and discuss the underlying spectral broadening processes from the femtosecond to the continuous wave regime. We also describe a new propagation model that integrates carrier dynamics.
\end{abstract}

\section{Introduction}

Since its first observation by Ranka et al. in 2000, supercontinuum (SC) generation in photonic crystal fiber (PCF) has been intensively studied over a wide range of experimental conditions [1, 2]. Nonlinear spectral broadening from self-phase modulation (SPM) plays a central role in SC generation, and its interaction with the novel fiber dispersion results in rich dynamics that are manifested in different ways depending on the particular parameter regime considered. Despite the complexity of these dynamics, numerical modeling has now resulted in a comprehensive physical understanding of the SC broadening mechanisms, and quantitative agreement between simulation and experiment has been obtained even for spectral bandwidths around an octave [3].

In this paper, we will present a synthetic review of the physics of SC generation in PCF as it is currently understood. In addition, we will discuss recent theoretical developments considering interaction regimes where the $\chi^{(3)}$ nonlinearity must be considered in a way that goes beyond a simple description involving only SPM. A particular result is the presentation of a new nonlinear propagation equation that extends the powerful concept of the pulse envelope to model dynamical effects on the underlying ultrafast carrier, and we use this model to examine the consequences for nonlinear propagation effects in PCF. The key features of our approach are a generalized nonlinear polarization term including third harmonic generation (THG), and the integration of carrier phase evolution effects onto the complex pulse envelope itself. Our extended envelope simulations are compared with the numerical integration of Maxwell's equations, and excellent quantitative agreement is obtained.

\section{Generalized Nonlinear Envelope Equation}

Our development follows the Blow-Wood time-domain formalism [4], but includes the full field $\chi^{(3)}$ nonlinearity, and uses a rigorous approach to forward and backward envelope factorization. Subject only to two approximations of (i) unidirectional propagation and (ii) a perturbative nonlinearity, we obtain a first-order propagation equation with no constraint on the temporal structure of the propagating envelope. This allows us to lift "slowly varying envelope" and "slowly evolving wave" approximations that have previously limited the application of envelope-approaches to sub-cycle regimes [5]. Specifically, writing the field $E(z, t)=\operatorname{Re}\left[A(z, t) \exp \left(-\mathrm{i} \omega_{0} t\right)\right]$, the propagation equation for $A(z, t)$ in the laboratory frame can be expressed as:

$$
\frac{\partial A}{\partial z}-\sum_{k \geq 0} \frac{i^{k+1}}{k !} \beta_{k} \frac{\partial^{k} A}{\partial t^{k}}=i \gamma\left(1+i \tau_{\text {shock }} \frac{\partial}{\partial t}\right)\left(\left(1-f_{R}\right)\left[|A|^{2}+\frac{1}{3} e^{-i 2 \omega_{0} t} A^{2}\right] A+f_{R} g(z, t, A)\right)
$$

where, on the right hand side, the term proportional to $|A|^{2} A$ describes SPM, the term proportional to $A^{3}$ describes THG, $f_{R}$ represents the fractional Raman contribution to the nonlinearity and $g(z, t, A)$ is a generalized Raman nonlinear response function [6]. This generalized envelope propagation equation allows for the accurate simulation of extreme nonlinear propagation phenomena whose study has previously been accessible only through the numerical solution of Maxwell's equations. 
To illustrate the success of Eq. (1) in modeling ultra-broadband nonlinear dynamics, we first consider the propagation of $5 \mathrm{fs}$ (FWHM) sech ${ }^{2}$ pulses of peak power $100 \mathrm{~kW}$ in an ideal dispersionless $\chi^{(3)}$ medium with nonlinearity $\gamma=0.68 \mathrm{~W}^{-1} \mathrm{~m}^{-1}$. The input wavelength is $830 \mathrm{~nm}$ and we consider propagation over $3.71 \mu \mathrm{m}$. Fig. 1(a) shows the output temporal characteristics obtained using numerical simulations based on Eq. (1), with the exploded view clearly illustrating the onset of a shock on the optical carrier. This shock arises from the presence of multiple higher-order harmonics, and these are apparent in the field spectrum that is also shown. Significantly, we have compared the temporal and spectral characteristics obtained from our generalized envelope Eq. (1) with simulations that directly integrate Maxwell's equations using the pseudospectral spatial domain (PSSD) method [7]. These results are shown as the open circles in Fig. 1(a). We stress the remarkable quantitative agreement that is obtained, even for this extreme case where spectral broadening extends over 10 times the carrier frequency, and the carrier exhibits shock dynamics on a sub-10 attosecond timescale. Additional simulations show similar agreement with increased propagation, and in the presence of dispersion.

These results confirm the validity of our generalized envelope model to describe ultra-broadband and sub-cycle propagation. We have thus used numerical simulations based on Eq. (1) to consider a fully realistic case in a $1 \mathrm{~mm}$ long silica tapered PCF nanowire of diameter $600 \mathrm{~nm}$. With parameters as above, but also including dispersion, Raman scattering, and the frequency dependence of the guided mode area, the results are shown in Fig. 1(b). The SC in this case spans over more than 2 octaves, significantly exceeding the range that has been successfully modeled in previous envelope-based numerical studies.

(a) Shock formation in dispersionless medium
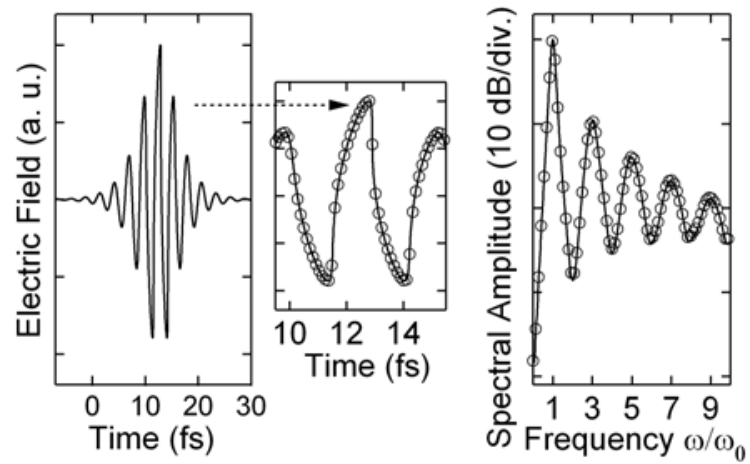

(b) SC generation in $1 \mathrm{~mm}$ long nanowire

Fig. 1 (a) Shock formation after $3.71 \mu \mathrm{m}$ propagation in a dispersionless $\chi^{(3)}$ medium. The left subfigure shows the temporal field obtained from the solution from the generalized nonlinear envelope equation, together with an exploded view comparing these envelope simulation results (solid line) with those obtained from Maxwell's equations (circles). The right subfigure compares the corresponding spectrum obtained using the generalized nonlinear envelope equation (solid line) with that from Maxwell's equations (circles). (b) Temporal and spectral characteristics obtained using Eq. (1) to model SC generation in a $1 \mathrm{~mm}$ nanowire of $600 \mathrm{~nm}$ diameter including dispersion, Raman scattering and modal effective area variation.

\section{Conclusions}

These results extend the use of an envelope description of pulse propagation into the sub-cycle regime, allowing the convenient modeling of carrier shocks and multi-octave SC generation. Significantly, additional simulations confirm the validity of this approach at all intensity levels less than the damage threshold of fused silica, and thus these results are particularly relevant to studying high intensity pulse propagation in PCF nanowires. We also suggest that these results could motivate a new generation of experiments studying nonlinear carrier shaping effects.

\section{References}

[1] J. K. Ranka, R. S. Windeler, A. J. Stentz, "Visible Continuum Generation in Air-Silica Microstructure Optical Fibers with Anomalous Dispersion at $800 \mathrm{~nm}$,” Opt. Lett. 25, 25-27 (2000).

[2] J. M. Dudley, G. Genty, S. Coen "Supercontinuum Generation in Photonic Crystal Fiber”, Rev. Mod. Phys. 78 1135-1184 (2006)

[3] K. L. Corwin, N. R. Newbury, J. M. Dudley, S. Coen, S. A. Diddams, K. Weber, and R. S. Windeler, "Fundamental Noise Limitations to Supercontinuum Generation in Microstructure Fiber,” Phys. Rev. Lett. 90, 113904 (2003).

[4] K. J. Blow and D. Wood, “Theoretical description of transient stimulated Raman scattering in optical fibers,” IEEE J. Quant. Electron. 25, 2665-2673 (1989).

[5] T. Brabec and F. Krausz, "Nonlinear optical pulse propagation in the single-cycle regime,” Phys. Rev. Lett. 78, 3282-3285 (1997).

[6] N. Karasawa , S. Nakamura, N. Nakagawa, M. Shibata, R. Morita, H. Shigekawa, and M. Yamashita, "Comparison between theory and experiment of nonlinear propagation for a-few-cycle and ultrabroadband optical pulses in a fused silica fiber,” IEEE J. Quantum Electron. 37, 398-404 (2001).

[7] J. C. A. Tyrrell, P. Kinsler, and G. H. C. New, "Pseudospectral spatial-domain: A new method for nonlinear pulse propagation in the fewcycle regime with arbitrary dispersion,” J. Mod. Opt. 52, 973-986 (2005). 